\title{
AN INEQUALITY FOR THE MODULUS OF THE RATIO OF TWO COMPLEX GAMMA FUNCTIONS
}

\author{
I. BELOVAS AND L. SAKALAUSKAS
}

Received 02 March, 2018

\begin{abstract}
The Euler gamma function is closely connected with the theory of zeta-functions. We prove a new inequality for the modulus of the ratio of two complex gamma functions $\Gamma(s) / \Gamma(2-$ $s$ ), arising in problems of the size of Selberg zeta-functions at places symmetric with respect to the critical line. This inequality, used together with technics of estimation, allows us in a different way re-prove and extend the result of R. Garunkštis and A. Grigutis for the modified Selberg zeta-function.
\end{abstract}

2010 Mathematics Subject Classification: 30A10; 33B15; 11M36

Keywords: Gamma function, Selberg zeta-function, ratios of complex functions, inequalities

\section{INTRODUCTION}

The Euler gamma function is closely connected with zeta-functions, and its properties are of great importance to the theory of zeta-functions and applications. E.g., the Riemann zeta-function satisfies the well-known functional equation [3]

$$
\zeta(s)=2^{s} \pi^{s-1} \sin \frac{\pi s}{2} \Gamma(1-s) \zeta(1-s),
$$

the Selberg zeta-function associated with the modular group $\operatorname{PSL}(2, \mathbb{Z})$ satisfies the functional equation [4]

$$
\begin{aligned}
& Z_{\mathrm{PSL}(2, \mathbb{Z})}(s)=Z_{\mathrm{PSL}(2, \mathbb{Z})}(1-s) \frac{\zeta(2 s)}{\zeta(2(1-s))} \frac{\Gamma(2 s)}{\Gamma(2(1-s))}(2 \pi)^{1-2 s} \times \\
& \times \exp \left(\frac{\pi}{3} \int_{0}^{s-1 / 2} v \tan \pi v d v-\frac{\pi}{2} \int_{0}^{s-1 / 2} \frac{d v}{\cos \pi v}-\frac{4 \pi}{3 \sqrt{3}} \int_{0}^{s-1 / 2} \frac{\cos \pi v / 3}{\cos \pi v} \mathrm{~d} v\right) .
\end{aligned}
$$

It is known [1] that

$$
|\zeta(1-s)|>|\zeta(s)|
$$

where $s=\sigma+i t$, is true for $\sigma>1 / 2$ and $t \geqslant 6.8$ except where $\zeta(s)=0$ (note that, if the inequality is valid without exceptions, then the Riemann hypothesis is true and vice versa). 
In [2] R. Garunkštis and A. Grigutis have proved a similar theorem for the modified Selberg zeta-function

$$
W(s)=Z_{\mathrm{PSL}(2, \mathbb{Z})}(s) / \zeta(2 s) .
$$

Theorem 1 (R. Garunkštis and A. Grigutis). If $1 / 2<\sigma<1$ and $t \geqslant 6.053$, then

$$
|W(1-s)|>|W(s)| .
$$

In the proof of the theorem R. Garunkštis and A. Grigutis established and used two lemmas for ratios of complex gamma functions [2].

Lemma 1 (R. Garunkštis and A. Grigutis). For $t \in \mathbb{R}$ the following inequality holds:

$$
\left|\frac{\Gamma(2+i t)}{\Gamma(i t)}\right|<\left|\frac{\sqrt{2}}{2}+i t\right|^{2} .
$$

Lemma 2 (R. Garunkštis and A. Grigutis). For $1 / 2<\sigma<1$ and $t \in \mathbb{R}$ the following inequality holds:

$$
\left|\frac{\Gamma(2 s)}{\Gamma(2(1-s))}\right| \leqslant\left|2 s-2-\frac{\sqrt{2}}{2}\right|^{4(\sigma-1 / 2)} .
$$

However, in order to obtain more subtle results, the following lemma has to be proved.

Lemma 3. Let $s=\sigma+i$. For $1<\sigma<2$ we have

$$
\left|\frac{\Gamma(s)}{\Gamma(2-s)}\right| \leqslant|s|^{2(\sigma-1)} .
$$

We can prove this statement using the theorem of Phragmén and Lindelöf.

Theorem 2 (Phragmén-Lindelöf). Let $f(z)$ be analytic in the strip

$$
S(\alpha, \beta)=\{z \mid z=x+i y, \alpha<x<\beta\} .
$$

Let us assume $|f(z)| \leqslant 1$ on the boundaries $x=\alpha$ and $x=\beta$, and moreover

$$
|f(z)|<C e^{e^{k|y|}}
$$

for some $C>0$ and $0<k<\frac{\pi}{\beta-\alpha}$. Then $|f(z)| \leqslant 1$ throughout the strip $S(\alpha, \beta)$.

Proof. See Rademacher [5] for the proof. 


\section{PRoof of LemMa 3}

Proof. Let $s=2-w, \sigma=2-\rho$. Then the statement of the theorem is equivalent to

$$
\left|\frac{\Gamma(2-w)}{\Gamma(w)}\right| \leqslant|2-w|^{2(1-\rho)},
$$

here $0<\rho<1$. Let us denote

$$
f(w)=\frac{\Gamma(2-w)}{\Gamma(w)(2-w)^{2(1-\rho)}} .
$$

First consider the left boundary $\rho=0, w=-i t$. We obtain

$$
f(w)=\frac{\Gamma(2+i t)}{\Gamma(-i t)(2+i t)^{2}} .
$$

Hence, since $\overline{\Gamma(z)}=\Gamma(\bar{z})$,

$$
\begin{aligned}
|f(w)| & =\frac{|\Gamma(2+i t)|}{|\Gamma(i t)||2+i t|^{2}}=\frac{|(1+i t)(i t)|}{4+t^{2}}= \\
& =\frac{\sqrt{t^{4}+t^{2}}}{t^{2}+4}=\sqrt{\frac{t^{4}+t^{2}}{t^{4}+8 t^{2}+16}},
\end{aligned}
$$

yielding us $|f(w)|<1$.

Now consider the right boundary $\rho=1, w=1-i t$. We obtain

$$
f(w)=\frac{\Gamma(1+i t)}{\Gamma(1-i t)} .
$$

Hence,

$$
|f(w)|=\frac{|\Gamma(1+i t)|}{|\Gamma(1-i t)|}=1 .
$$

Next, let us consider the modulus of the function $f(w)$. Since $|\Gamma(z)| \leqslant \Gamma(\Re z)$ and $\Gamma(2-\rho)=\Gamma(\sigma) \leqslant 1$, we obtain

$$
|f(w)|=\frac{|\Gamma(2-w)|}{|\Gamma(w)||2-w|^{2(1-\rho)}} \leqslant \frac{\Gamma(2-\rho)}{\left((2-\rho)^{2}+t^{2}\right)^{(1-\rho)}}\left|\frac{1}{\Gamma(w)}\right| \leqslant\left|\frac{1}{\Gamma(w)}\right| .
$$

It is known [5], that if $t$ is sufficiently large (i.e. $|t| \geqslant 1$ ), then the reciprocal gamma function

$$
\frac{1}{\Gamma(w)}=O\left(e^{\frac{\pi}{2}|t|}|t|^{\frac{1}{2}-\rho}\right)
$$

Since the reciprocal gamma function is an entire function, it is bounded in every compact subset of the complex plane (in particular, for $0 \leqslant \rho \leqslant 1$ and $0 \leqslant t \leqslant 1$ the modulus of the reciprocal gamma function $|1 / \Gamma(w)| \leqslant 2)$.

Thus, $|f(w)|=O\left(e^{e^{|t|}}\right)$ for $t \in \mathbb{R}$, which yields us the statement of Lemma 3 . 


\section{THEOREM FOR THE MODIFIED SELBERG ZETA-FUNCTION}

The inequality (1.7) of Lemma 3, used together with technics of estimation, allows us (see Theorem 3) in a different way re-prove and extend the result of R. Garunkštis and A. Grigutis for the modified Selberg zeta-function (cf. Theorem 1).

Theorem 3. For $1 / 2<\sigma<1$ and $t \in\left[0, t_{1}\right) \cup\left(t_{2}, \infty\right)$ we have

$$
|W(1-s)|>|W(s)| \text {. }
$$

Here $t_{1}=1.740440 \ldots$ and $t_{2}=6.088036 \ldots$ are the roots of the function

$$
L_{1}(t)=\log \left(\frac{1+t^{2}}{\pi \cosh \log \left(2 \sinh \frac{\pi t}{6}\right)}\right) .
$$

By the definition of the modified Selberg zeta-function (1.3) and Lemma 3 we have

$$
\left|\frac{W(s)}{W(1-s)}\right|=\left|\frac{\Gamma(2 s)}{\Gamma(2(1-s))}(2 \pi)^{1-2 s} e^{Q(s)}\right| \leqslant\left(\frac{|2 s|^{2}}{2 \pi}\right)^{2 \sigma-1} e^{\Re(Q(s))},
$$

here

$$
Q(s)=\int_{0}^{s-1 / 2} \frac{\pi}{3} v \tan \pi v-\frac{\pi}{2 \cos \pi v}-\frac{4 \pi}{3 \sqrt{3}} \frac{\cos \pi v / 3}{\cos \pi v} \mathrm{~d} v .
$$

Integral (3.4) can be evaluated using triangular contour with vertices at $A(0,0), B(\sigma-$ $1 / 2, t)$ and $C(\sigma-1 / 2,0): \int_{A C}+\int_{C B}+\int_{B A}=0$. Hence,

$$
\underbrace{\Re(Q(s))}_{\Re \int_{A B}}=\underbrace{I_{1}(\sigma)}_{\int_{A C}}+\underbrace{R(\sigma, t)}_{\Re \int_{C B}} \text {. }
$$

Here

$$
I_{1}(\sigma)=\int_{0}^{\sigma-1 / 2} \frac{\pi}{3} \theta \tan \pi \theta-\frac{\pi}{2 \cos \pi \theta}-\frac{4 \pi}{3 \sqrt{3}} \frac{\cos \pi \theta / 3}{\cos \pi \theta} \mathrm{d} \theta
$$

and

$$
R(\sigma, t)=\Re\left\{\int_{0}^{t} \frac{\frac{\pi i}{3}\left(\sigma-\frac{1}{2}+i \theta\right)}{\cot \left(\pi\left(\sigma-\frac{1}{2}+i \theta\right)\right)}-\frac{\frac{\pi i}{2}+\frac{4 \pi i}{3 \sqrt{3}} \cos \left(\frac{\pi}{3}\left(\sigma-\frac{1}{2}+i \theta\right)\right)}{\cos \left(\pi\left(\sigma-\frac{1}{2}+i \theta\right)\right)} \mathrm{d} \theta\right\} .
$$

Let us denote

$$
L(\sigma, t)=(2 \sigma-1) \log \frac{\sigma^{2}+t^{2}}{\pi / 2}+I_{1}(\sigma)+R(\sigma, t) .
$$

Hence (cf. (3.3) and (3.5)),

$$
\log \left|\frac{W(s)}{W(1-s)}\right| \leqslant L(\sigma, t)
$$


Calculating function $I_{1}(\sigma)$ (3.6), we obtain

$$
\begin{aligned}
I_{1}(\sigma) & =\int_{0}^{\sigma-1 / 2} \frac{\pi}{3} \theta \tan \pi \theta-\frac{\pi}{2 \cos \pi \theta}-\frac{4 \pi}{3 \sqrt{3}} \frac{\cos \pi \theta / 3}{\cos \pi \theta} \mathrm{d} \theta= \\
& =-\frac{1}{6 \pi} \mathrm{Cl}_{2}(2 \pi \sigma)-\frac{2 \sigma-1}{6} \log 2-\frac{2 \sigma-1}{6} \log \sin \pi \sigma- \\
& -\frac{1}{2} \log \tan \frac{\pi \sigma}{2}+\frac{2}{3} \log \left(\frac{\sqrt{3}}{2} \cot \frac{\pi \sigma}{3}-\frac{1}{2}\right) .
\end{aligned}
$$

Here $\mathrm{Cl}_{2}(x)$ is the Clausen function of order 2,

$$
\mathrm{Cl}_{2}(x)=-\int_{0}^{x} \log \left|2 \sin \frac{t}{2}\right| \mathrm{d} t .
$$

Noticing that (cf. (3.7))

$$
\begin{gathered}
\Re\left\{\frac{i(\sigma-1 / 2+i \theta)}{\cot (\pi(\sigma-1 / 2+i \theta))}\right\}=\frac{\theta \sin 2 \pi \sigma+(1 / 2-\sigma) \sinh 2 \pi \theta}{\cosh 2 \pi \theta-\cos 2 \pi \sigma}, \\
\Re\left\{\frac{i / 2}{\cos (\pi(\sigma-1 / 2+i \theta))}\right\}=\frac{\cos \pi \sigma \sinh \pi \theta}{\cosh 2 \pi \theta-\cos 2 \pi \sigma}
\end{gathered}
$$

and

$$
\Re\left\{\frac{i \cos \left(\frac{\pi}{3}\left(\sigma-\frac{1}{2}+i \theta\right)\right)}{\cos \left(\pi\left(\sigma-\frac{1}{2}+i \theta\right)\right)}\right\}=\frac{\cos \left(\frac{2 \pi \sigma}{3}+\frac{\pi}{6}\right) \sinh \frac{4 \pi \theta}{3}+\cos \left(\frac{4 \pi \sigma}{3}-\frac{\pi}{6}\right) \sinh \frac{2 \pi \theta}{3}}{\cosh 2 \pi \theta-\cos 2 \pi \sigma} .
$$

we calculate function $R(\sigma, t)$ (3.7), obtaining

$$
\begin{aligned}
R(\sigma, t)= & \underbrace{\frac{\pi}{3} \sin 2 \pi \sigma \int_{0}^{t} \frac{\theta}{\cosh 2 \pi \theta-\cos 2 \pi \sigma} \mathrm{d} \theta}_{=I_{2}(\sigma, t)}+ \\
& +\underbrace{\frac{\pi}{3}\left(\frac{1}{2}-\sigma\right) \int_{0}^{t} \frac{\sinh 2 \pi \theta}{\cosh 2 \pi \theta-\cos 2 \pi \sigma} \mathrm{d} \theta}_{=I_{3}(\sigma, t)}+ \\
& +\underbrace{\pi \cos \pi \sigma \int_{0}^{t} \frac{-\sinh \pi \theta}{\cosh 2 \pi \theta-\cos 2 \pi \sigma} \mathrm{d} \theta}_{=I_{5}(\sigma, t)}+ \\
& +\underbrace{\frac{-4 \pi}{3 \sqrt{3}} \int_{0}^{t} \frac{\cos \left(\frac{2 \pi \sigma}{3}+\frac{\pi}{6}\right) \sinh \frac{4 \pi \theta}{3}+\cos \left(\frac{4 \pi \sigma}{3}-\frac{\pi}{6}\right) \sinh \frac{2 \pi \theta}{3}}{\cosh 2 \pi \theta-\cos 2 \pi \sigma} \mathrm{d} \theta}_{=I_{4}(\sigma, t)} .
\end{aligned}
$$


Note that $R(\sigma, t)$ is even function by $t$, thus, it suffices to consider non-negative $t$ values. Calculating summands of the function $R(\sigma, t)(3.15)$, we obtain

$$
\begin{aligned}
I_{2}(\sigma, t)= & \frac{\pi}{3} \sin 2 \pi \sigma \int_{0}^{t} \frac{\theta}{\cosh 2 \pi \theta-\cos 2 \pi \sigma} \mathrm{d} \theta, \\
I_{3}(\sigma, t)= & -\frac{2 \sigma-1}{12} \log (\cosh 2 \pi t-\cos 2 \pi \sigma)+ \\
& +\frac{2 \sigma-1}{6} \log \sin \pi \sigma+\frac{2 \sigma-1}{12} \log 2, \\
I_{4}(\sigma, t)= & \frac{1}{2} \log \tan \frac{\pi \sigma}{2}-\frac{1}{4} \log \frac{\cosh \pi t-\cos \pi \sigma}{\cosh \pi t+\cos \pi \sigma}, \\
I_{5}(\sigma, t)= & -\frac{2}{3} \log \left(\frac{\sqrt{3}}{2} \cot \frac{\pi \sigma}{3}-\frac{1}{2}\right)- \\
& -\frac{1}{3} \log \frac{\cosh \frac{2 \pi t}{3}-\cos \frac{2 \pi \sigma}{3}}{\cosh \frac{2 \pi t}{3}-\cos \frac{2 \pi(\sigma-1)}{3}} .
\end{aligned}
$$

Expressions (3.16)-(3.19) allow us to calculate function $L(\sigma, t)(3.8)$,

$$
\begin{aligned}
L(\sigma, t)= & (2 \sigma-1) \log \left(\frac{2}{\pi}\left(\sigma^{2}+t^{2}\right)\right)-(2 \sigma-1) \frac{\log 2}{12}- \\
& -\frac{1}{6 \pi} \mathrm{Cl}_{2}(2 \pi \sigma)+\frac{\pi}{3} \sin 2 \pi \sigma \int_{0}^{t} \frac{\theta}{\cosh 2 \pi \theta-\cos 2 \pi \sigma} \mathrm{d} \theta- \\
& -\frac{2 \sigma-1}{12} \log (\cosh 2 \pi t-\cos 2 \pi \sigma)+\frac{1}{4} \log \frac{\cosh \pi t+\cos \pi \sigma}{\cosh \pi t-\cos \pi \sigma}+ \\
& +\frac{1}{3} \log \frac{\cosh \frac{2 \pi t}{3}-\cos \frac{2 \pi(\sigma-1)}{3}}{\cosh \frac{2 \pi t}{3}-\cos \frac{2 \pi \sigma}{3}} .
\end{aligned}
$$

Next we will establish several auxiliary lemmas concerning the behaviour of the function $L(\sigma, t)$.

\section{THE DERIVATIVES OF THE FUNCTION $L(\sigma, t)$}

Lemma 4. For $1 / 2<\sigma<1$ and fixed $1 / 2 \leqslant t<\infty$ the function $L(\sigma, t)(3.20)$ is convex by $\sigma$. 
Proof. Let us calculate partial derivatives of the function $L(\sigma, t)$ with respect to the variable $\sigma$,

$$
\begin{aligned}
& L_{\sigma}^{\prime}(\sigma, t)=\underbrace{2 \log \frac{\sigma^{2}+t^{2}}{\pi / 2}}_{=F_{1}(\sigma, t)}+\underbrace{\frac{2 \sigma(2 \sigma-1)}{\sigma^{2}+t^{2}}}_{=F_{2}(\sigma, t)}+\underbrace{\frac{-\pi t}{3} \frac{\sinh 2 \pi t}{\cosh 2 \pi t-\cos 2 \pi \sigma}}_{=F_{3}(\sigma, t)}+ \\
& +\underbrace{\frac{\pi}{6}(2 \sigma-1) \frac{-\sin 2 \pi \sigma}{\cosh 2 \pi t-\cos 2 \pi \sigma}}_{=F_{4}(\sigma, t)}+\underbrace{\frac{-\pi \sin \pi \sigma \cosh \pi t}{\cosh 2 \pi t-\cos 2 \pi \sigma}}_{=F_{5}(\sigma, t)}+ \\
& +\underbrace{\frac{\pi}{3 \sqrt{3}} \frac{1-2 \cosh \frac{2 \pi t}{3} \cos \left(\frac{2 \pi \sigma}{3}-\frac{\pi}{3}\right)}{\left(\cosh \frac{2 \pi t}{3}-\cos \frac{2 \pi(\sigma-1)}{3}\right)\left(\cosh \frac{2 \pi t}{3}-\cos \frac{2 \pi \sigma}{3}\right)} .}_{=F_{6}(\sigma, t)} \\
& L_{\sigma \sigma}^{\prime \prime}(\sigma, t)=\frac{12 \sigma-2}{\sigma^{2}+t^{2}}-\frac{4 \sigma^{2}(2 \sigma-1)}{\left(\sigma^{2}+t^{2}\right)^{2}}+ \\
& +\underbrace{\frac{-\pi}{3} \frac{\sin 2 \pi \sigma+\pi(2 \sigma-1) \cos 2 \pi \sigma+3 \pi \cos \pi \sigma \cosh \pi t}{\cosh 2 \pi t-\cos 2 \pi \sigma}}_{=G_{1}(\sigma, t)}+ \\
& +\underbrace{\frac{\pi^{2}}{3} \sin 2 \pi \sigma \frac{(2 \sigma-1) \sin 2 \pi \sigma+6 \sin \pi \sigma \cosh \pi t+2 t \sinh 2 \pi t}{(\cosh 2 \pi t-\cos 2 \pi \sigma)^{2}}}_{=G_{2}(\sigma, t)}+ \\
& +\underbrace{\frac{2 \pi^{2}}{9 \sqrt{3}} \frac{\sin \left(\frac{2 \pi \sigma}{3}-\frac{\pi}{3}\right)}{\left(\cosh \frac{2 \pi t}{3}-\cos \frac{2 \pi(\sigma-1)}{3}\right)^{2}\left(\cosh \frac{2 \pi t}{3}-\cos \frac{2 \pi \sigma}{3}\right)^{2}} \times}_{=G_{3}(\sigma, t)} \\
& \times \underbrace{\left(2 \cos \left(\frac{2 \pi \sigma}{3}-\frac{\pi}{3}\right)-\cosh \frac{2 \pi t}{3}\left(\frac{7}{2}+\cos \left(\frac{4 \pi \sigma}{3}-\frac{2 \pi}{3}\right)\right)+2 \cosh ^{3} \frac{2 \pi t}{3}\right)}_{=G_{4}(\sigma, t)} .
\end{aligned}
$$

First consider the interval $1 / 2 \leqslant t \leqslant 2$. Let us give a lower bounds of the functions $G_{k}(\sigma, t)$, which are defined in (4.2). Denote

$$
\begin{aligned}
& v_{1}(\sigma, t)=\cosh 2 \pi t-\cos 2 \pi \sigma \\
& u_{1}(\sigma, t)=\sin 2 \pi \sigma+\pi(2 \sigma-1) \cos 2 \pi \sigma+3 \pi \cos \pi \sigma \cosh \pi t .
\end{aligned}
$$

Note that $v_{1}(\sigma, t)$ is positive for $t \in \mathbb{R}$ and $1 / 2<\sigma<1$. Next, $u_{1}(\sigma, t)$ is negative for $t \in \mathbb{R}$ and $1 / 2<\sigma \leqslant 3 / 4$. For $3 / 4<\sigma<1$, we have $0<\cos 2 \pi \sigma<1$ and $\cos \pi \sigma<0$. 
Hence,

$$
u_{1}(\sigma, t) \leqslant \underbrace{\sin 2 \pi \sigma+\pi(2 \sigma-1)+3 \pi \cos \pi \sigma}_{=w_{1}(\sigma)} .
$$

The function $w_{1}(\sigma)$ is convex, because

$$
w_{1}^{\prime \prime}(\sigma)=-4 \pi^{2} \underbrace{\sin 2 \pi \sigma}_{\leqslant 0}-3 \pi^{3} \underbrace{\cos \pi \sigma}_{\leqslant 0}>0,
$$

for $3 / 4<\sigma<1$. With $w_{1}(3 / 4)<0$ and $w_{1}(1)<0$ it yields us $u_{1}(\sigma, t)<0$ for $t \in \mathbb{R}$ and $1 / 2<\sigma<1$. Thus,

$$
G_{1}(\sigma, t)=-\frac{\pi}{3} \frac{u_{1}(\sigma, t)}{v_{1}(\sigma, t)}>0
$$

for $t \in \mathbb{R}$ and $1 / 2<\sigma<1$.

The function

$$
G_{2}(\sigma, t)>\underbrace{\frac{2 \pi^{2}}{-3} \frac{(3+2 t \sinh \pi t) \cosh \pi t}{(\cosh 2 \pi t-1)^{2}}}_{=u_{2}(t) \text {-increasing }} \geqslant u_{2}(1 / 2)>\varepsilon=-0.8,
$$

for $t \geqslant 1 / 2$ and $1 / 2<\sigma<1$.

The function $G_{3}(\sigma, t)$ is positive for $1 / 2<\sigma<1$.

Next let us show that $G_{4}(\sigma, t)$ is increasing by $\sigma$ and by $t$. Indeed, consider the derivatives

$$
\begin{aligned}
\left(G_{4}\right)_{\sigma}^{\prime} & =-\frac{4 \pi}{3} \sin \left(\frac{2 \pi \sigma}{3}-\frac{\pi}{3}\right)+\frac{4 \pi}{3} \cosh \frac{2 \pi t}{3} \sin \left(\frac{4 \pi \sigma}{3}-\frac{2 \pi}{3}\right)= \\
& =\frac{4 \pi}{3} \underbrace{\sin \left(\frac{2 \pi \sigma}{3}-\frac{\pi}{3}\right)}_{>0}(\underbrace{2 \cos \left(\frac{2 \pi \sigma}{3}-\frac{\pi}{3}\right)}_{\in(1,2)} \underbrace{\left.\cosh \frac{2 \pi t}{3}-1\right)>0 .}_{\geqslant 1} \\
\left(G_{4}\right)_{t}^{\prime} & =\frac{2 \pi}{3} \sinh ^{\frac{2 \pi t}{3}}\left(6 \cosh ^{2} \frac{2 \pi t}{3}-\left(\frac{7}{2}+\cos \left(\frac{4 \pi \sigma}{3}-\frac{2 \pi}{3}\right)\right)\right)>0
\end{aligned}
$$

for $t>0$. Thus $G_{4}(\sigma, t) \geqslant G_{4}(1 / 2,1 / 2)>0$. Hence,

$$
L_{\sigma \sigma}^{\prime \prime}(\sigma, t) \geqslant \underbrace{\frac{12 \sigma-2}{\sigma^{2}+t^{2}}-\frac{4 \sigma^{2}(2 \sigma-1)}{\left(\sigma^{2}+t^{2}\right)^{2}}-0.8}_{=B(\sigma, t)} .
$$


Note that the function $B(\sigma, t)$ is decreasing by $t$. Consider

$$
\begin{aligned}
B_{t}^{\prime}(\sigma, t) & =(12 \sigma-2) \frac{-2 t}{\left(\sigma^{2}+t^{2}\right)^{2}}+\left(8 \sigma^{3}-4 \sigma^{2}\right) \frac{4 t}{\left(\sigma^{2}+t^{2}\right)^{3}}= \\
& =\frac{4 t}{\left(\sigma^{2}+t^{2}\right)^{3}}(\underbrace{(2 \sigma-3)}_{<0} \sigma^{2}-(6 \sigma-1) t^{2})<0
\end{aligned}
$$

for $t>0$. Thus, $B(\sigma, t) \geqslant B(\sigma, 2)=B_{2}(\sigma)$ and

$$
B_{2}(\sigma)=\frac{12 \sigma-2}{\sigma^{2}+4}-\frac{4 \sigma^{2}(2 \sigma-1)}{\left(\sigma^{2}+4\right)^{2}}-0.8=4 \frac{\sigma^{3}(1-0.2 \sigma)+\left(12 \sigma-1.1 \sigma^{2}-5.2\right)}{\left(\sigma^{2}+4\right)^{2}}>0
$$

for $1 / 2<\sigma<1$, yielding us the statement of the lemma for $1 / 2 \leqslant t \leqslant 2$.

Next consider the interval $t \geqslant C$. Let $C=\sqrt{\frac{1}{2}+\sqrt{\frac{11}{12}}}=1.20724 \ldots$. Let us consider the second partial derivative $L_{\sigma \sigma}^{\prime \prime}$ (4.2). We have shown that the function $G_{1}(\sigma, t)(4.3)$ and the product $G_{3}(\sigma, t) G_{4}(\sigma, t)$ are positive for $t>0$. Hence, using (4.4), we obtain

$$
L_{\sigma \sigma}^{\prime \prime}(\sigma, t) \geqslant \underbrace{\frac{12 \sigma-2}{\sigma^{2}+t^{2}}-\frac{4 \sigma^{2}(2 \sigma-1)}{\left(\sigma^{2}+t^{2}\right)^{2}}-\frac{\pi^{2}}{6} \frac{(3+2 t \sinh \pi t) \cosh \pi t}{\sinh ^{4} \pi t}}_{=D(\sigma, t)} .
$$

Let us show that $D(\sigma, t)$ is increasing by $\sigma$. Indeed, consider the derivative

$$
D_{\sigma}^{\prime}(\sigma, t)=4 \frac{P(\sigma, t)}{\left(\sigma^{2}+t^{2}\right)^{3}}
$$

here

$$
P(\sigma, t)=-\sigma^{4}-\sigma^{3}-6 t^{2} \sigma^{2}+3 t^{2} \sigma+3 t^{4} .
$$

For $1 / 2<\sigma<1$ the polynomial $P(\sigma, t)$ is concave by $\sigma$, because the second derivative

$$
P_{\sigma \sigma}^{\prime \prime}=-12 \sigma^{2}-6 \sigma-12 t^{2}<0 .
$$

The function is nonnegative at the endpoints of the interval,

$$
P(1 / 2, t)=3 t^{4}-3 / 16 \geqslant 0
$$

for $t \geqslant 1 / 2$, and

$$
P(1, t)=3 t^{4}-3 t^{2}-2 \geqslant 0
$$


for $t \geqslant C$. Hence, $P(\sigma, t) \geqslant 0$ and $D_{\sigma}^{\prime}(\sigma, t) \geqslant 0$ for $t \geqslant C$, yielding us $D(\sigma, t)>$ $D(1 / 2, t)$. Consider

$$
\begin{aligned}
D(1 / 2, t) & =\frac{4}{t^{2}+1 / 4}-\frac{\pi^{2}}{6} \frac{(3+2 t \sinh \pi t) \cosh \pi t}{\sinh ^{4} \pi t}= \\
& =\frac{4}{t^{2}} \frac{1}{1+1 /\left(4 t^{2}\right)}-\frac{4 \pi^{2}}{3} t e^{-2 \pi t} \frac{\left(1+3 e^{-\pi t} / t-e^{-2 \pi t}\right)\left(1+e^{-2 \pi t}\right)}{\left(1-e^{-2 \pi t}\right)^{4}} \geqslant \\
& \geqslant \frac{4 H}{t^{2}}-4 A t e^{-2 \pi t}=\frac{4}{t^{2}} \underbrace{\left(H-A t^{3} e^{-2 \pi t}\right)}_{=E(t)} .
\end{aligned}
$$

Here

$H=\frac{1}{1+1 /\left(4 C^{2}\right)}=0.854 \ldots, \quad A=\frac{\pi^{2}}{3} \frac{\left(1+3 e^{-\pi C} / C\right)\left(1+e^{-2 \pi C}\right)}{\left(1-e^{-2 \pi C}\right)^{4}}=3.483 \ldots$

The function $E(t)$ is increasing for $t \geqslant C$ and $E(C)$ is positive, yielding us the statement of the lemma.

Lemma 5. For $1 / 2<\sigma<1$, the derivative $L_{t}^{\prime}(\sigma, t)$

(1) is positive for $t \in(0,3.53]$,

(2) is negative for $t \in[3.77, \infty)$.

Proof. Let us calculate the first partial derivative

$$
\begin{aligned}
L_{t}^{\prime}(\sigma, t)= & \underbrace{(2 \sigma-1) \frac{2 t}{\sigma^{2}+t^{2}}}_{=N_{1}(\sigma, t)}+\underbrace{\frac{\pi}{3} \frac{t \sin 2 \pi \sigma}{\cosh 2 \pi t-\cos 2 \pi \sigma}}_{=N_{2}(\sigma, t)}+ \\
& +\underbrace{\frac{\pi}{6}(2 \sigma-1) \frac{-\sinh 2 \pi t}{\cosh 2 \pi t-\cos 2 \pi \sigma}}_{=N_{3}(\sigma, t)}+\underbrace{\frac{-\pi \cos \pi \sigma \sinh \pi t}{\cosh 2 \pi t-\cos 2 \pi \sigma}}_{=N_{4}(\sigma, t)}+ \\
& +\underbrace{\frac{2 \pi}{9} \frac{\sinh \frac{2 \pi t}{3}\left(\cos \frac{2 \pi(\sigma-1)}{3}-\cos \frac{2 \pi \sigma}{3}\right)}{\left(\cosh \frac{2 \pi t}{3}-\cos \frac{2 \pi(\sigma-1)}{3}\right)\left(\cosh \frac{2 \pi t}{3}-\cos \frac{2 \pi \sigma}{3}\right)}}_{=N_{5}(\sigma, t)} .
\end{aligned}
$$

Estimating functions in (4.5) we obtain $N_{5}(\sigma, t)>0, N_{1}(\sigma, t)>0$. Now let us show that $N_{2}(\sigma, t)+N_{3}(\sigma, t)+N_{4}(\sigma, t)>0$. It is sufficient to prove that

$$
\underbrace{\frac{1}{3} t \sin 2 \pi \sigma-\frac{2 \sigma-1}{6} \sinh 2 \pi t-\cos \pi \sigma \sinh \pi t}_{=N(\sigma, t)}>0 .
$$


Consider the derivative

$$
\begin{aligned}
N_{t}^{\prime}(\sigma, t) & =\frac{1}{3} \sin 2 \pi \sigma-\frac{2 \sigma-1}{3} \pi \cosh 2 \pi t-\pi \cos \pi \sigma \cosh \pi t= \\
& =-\frac{1}{3}\left(2 \pi(2 \sigma-1) \cosh ^{2} \pi t+3 \pi \cos \pi \sigma \cosh \pi t-(\sin 2 \pi \sigma+(2 \sigma-1) \pi)\right) .
\end{aligned}
$$

The positive root of the quadratic equation

$$
r(\sigma)=\frac{-3 \pi \cos \pi \sigma+\sqrt{9 \pi^{2} \cos ^{2} \pi \sigma+8 \pi(2 \sigma-1) \sin 2 \pi \sigma+8 \pi^{2}(2 \sigma-1)^{2}}}{4 \pi(2 \sigma-1)} .
$$

For $1 / 2<\sigma<1$,

For $0<t \leqslant 0.37$

$$
\frac{3+\sqrt{17}}{4}<r(\sigma)<\frac{3 \pi}{4}
$$

$$
1<\cosh 2 \pi t \leqslant 1.755<\frac{3+\sqrt{17}}{4} .
$$

Hence, $N_{t}^{\prime}(\sigma, t)>0$ and $N(\sigma, t)>N(\sigma, 0)=0$, yielding us the statement of the lemma for $t \in(0,0.37]$.

Consider the first partial derivative in the interval $t \in[0.37,3.53]$,

$$
\begin{aligned}
L_{t}^{\prime}(\sigma, t)= & (2 \sigma-1) \frac{2 t}{\sigma^{2}+t^{2}}+\underbrace{\frac{\pi}{3} \frac{\cos \pi \sigma(2 t \sin \pi \sigma-3 \sinh \pi t)}{\cosh 2 \pi t-\cos 2 \pi \sigma}}_{=H_{2}(\sigma, t)}+ \\
& +\underbrace{\frac{\pi}{6}(2 \sigma-1) \frac{-\sinh 2 \pi t}{\cosh 2 \pi t-\cos 2 \pi \sigma}}_{=H_{3}(\sigma, t)}+ \\
& +\underbrace{\frac{2 \pi}{9} \frac{\sinh \frac{2 \pi t}{3}\left(\cos \frac{2 \pi(\sigma-1)}{3}-\cos \frac{2 \pi \sigma}{3}\right)}{\left(\cosh \frac{2 \pi t}{3}-\cos \frac{2 \pi(\sigma-1)}{3}\right)\left(\cosh \frac{2 \pi t}{3}-\cos \frac{2 \pi \sigma}{3}\right)}}_{=H_{4}(\sigma, t)} .
\end{aligned}
$$

Estimating $H_{2}(\sigma, t)$ and $H_{4}(\sigma, t)$ we obtain $H_{2}(\sigma, t)>0$ and $H_{4}(\sigma, t)>0$ for $1 / 2<$ $\sigma<1$. Thus,

$$
\begin{aligned}
L_{t}^{\prime}(\sigma, t) & >(2 \sigma-1) \frac{2 t}{\sigma^{2}+t^{2}}+\frac{\pi}{6}(2 \sigma-1) \frac{-\sinh 2 \pi t}{\cosh 2 \pi t-\cos 2 \pi \sigma}> \\
& >(2 \sigma-1)\left(\frac{2 t}{\sigma^{2}+t^{2}}-\frac{\pi}{6} \frac{\sinh 2 \pi t}{\cosh 2 \pi t-1}\right)= \\
& =(2 \sigma-1) \underbrace{\left(\frac{2 t}{\sigma^{2}+t^{2}}-\frac{\pi}{6} \operatorname{coth} \pi t\right)}_{=M(\sigma, t)} .
\end{aligned}
$$


The function $M(\sigma, t)$ is decreasing by $\sigma$, hence

$$
M(\sigma, t) \geqslant \frac{2 t}{1+t^{2}}-\frac{\pi}{6} \operatorname{coth} \pi t \geqslant \underbrace{\frac{2 t}{1+t^{2}}-\frac{\pi}{6} A_{k}}_{=M_{k}(t)} .
$$

Here

$$
A_{k}= \begin{cases}\operatorname{coth} 0.37 \pi & \text { for } 0.37 \leqslant t<2.77 \\ \operatorname{coth} 2.77 \pi & \text { for } 2.77 \leqslant t \leqslant 3.53\end{cases}
$$

Now

$$
M_{k}^{\prime}(t)=2 \frac{1-t^{2}}{\left(1+t^{2}\right)^{2}} .
$$

For $0.37 \leqslant t<2.77$ the function $M_{1}(t)$ increases in the interval $(0.37,1)$ and decreases in the interval $(1,2.77)$. At endpoints the function is positive, $M_{1}(0.37)>0$ and $M_{1}(2.77)>0$.

For $2.77 \leqslant t \leqslant 3.53$ the function $M_{2}(t)$ decreases. At the endpoint the function is positive, $M_{2}(3.53)>0$, yielding us the statement of the lemma for the interval $0.37 \leqslant t \leqslant 3.53$.

Estimating functions $N_{k}(\sigma, t)$ in (4.5) for $t \geqslant 3.77$ we obtain (note that $N_{2}(\sigma, t)<$ $0)$

$$
\begin{aligned}
L_{t}^{\prime}(\sigma, t) & <\frac{2 t(2 \sigma-1)}{\sigma^{2}+t^{2}}-\frac{\pi(2 \sigma-1)}{6}-\frac{\pi \cos \pi \sigma}{2 \sinh \pi t}+\frac{2 \pi}{3 \sqrt{3}} \frac{\operatorname{coth} \frac{\pi t}{3} \sin \left(\frac{2 \pi \sigma}{3}-\frac{\pi}{3}\right)}{\cosh \frac{2 \pi t}{3}-\cos \frac{2 \pi \sigma}{3}}< \\
& <(2 \sigma-1)(\frac{2 t}{\sigma^{2}+t^{2}}+\underbrace{\left(-\frac{\pi}{6}+\frac{\pi^{2}}{4 \sinh \pi t}+\frac{2 \pi^{2}}{\left.9 \sqrt{3} \frac{\operatorname{coth} \frac{\pi t}{3}}{\cosh \frac{2 \pi t}{3}-\frac{1}{2}}\right)}\right)}_{=K(\sigma, t)} .
\end{aligned}
$$

Consider the derivative of the function $\mu(t)$

$$
\mu^{\prime}(t)=\underbrace{\frac{\pi^{3}}{-4} \frac{\cosh \pi t}{\sinh ^{2} \pi t}}_{<0}+\underbrace{\frac{-2 \pi^{3}}{27 \sqrt{3}} \frac{\sinh ^{-2} \frac{\pi t}{3}\left(\cosh \frac{2 \pi t}{3}-\frac{1}{2}\right)+2 \operatorname{coth} \frac{\pi t}{3} \sinh \frac{2 \pi t}{3}}{\left(\cosh \frac{2 \pi t}{3}-\frac{1}{2}\right)^{2}}}_{<0} .
$$

Hence,

$$
K_{t}^{\prime}(\sigma, t)=(2 \sigma-1)(\underbrace{\frac{2\left(\sigma^{2}-t^{2}\right)}{\left(\sigma^{2}+t^{2}\right)^{2}}}_{<0}+\underbrace{\mu^{\prime}(t)}_{<0})<0
$$


the function $K(\sigma, t)$ is decreasing by $t$ and $K(\sigma, t) \leqslant K(\sigma, 3.77)<0$, yielding us the statement of the lemma.

\section{AuXiliary Lemmas}

Let us denote

$$
L_{1}(t)=L(1, t)
$$

Lemma 6. The function $L_{1}(t)$

(1) is negative for $t \in\left(0, t_{1}\right) \cup\left(t_{2}, \infty\right)$,

(2) is positive for $\left(t_{1}, t_{2}\right)$,

(3) has unique maximum point at $t^{*} \in\left(t_{1}, t_{2}\right)$.

Here $t_{1}=1.740440 \ldots$ and $t_{2}=6.088036 \ldots$ are the roots of the function.

Proof. By (5.1) and (3.20) we obtain

$$
\begin{aligned}
L_{1}(t) & =\log \left(\frac{2}{\pi}\left(1+t^{2}\right)\right)-\frac{\log 2}{12}-\frac{1}{12} \log (\cosh 2 \pi t-1)+ \\
& +\frac{1}{4} \log \frac{\cosh \pi t-1}{\cosh \pi t+1}+\frac{1}{3} \log \frac{\cosh \frac{2 \pi t}{3}-1}{\cosh \frac{2 \pi t}{3}-\frac{1}{2}}= \\
& =\log \left(\frac{1+t^{2}}{\pi} \frac{\sinh \frac{\pi t}{6}}{\sinh ^{2} \frac{\pi t}{6}+\left(\frac{1}{2}\right)^{2}}\right)=\log \left(\frac{1+t^{2}}{\pi \cosh \log \left(2 \sinh \frac{\pi t}{6}\right)}\right) .
\end{aligned}
$$

Note that

$$
\lim _{t \rightarrow 0+} L_{1}(t)=-\infty, \quad \quad \lim _{t \rightarrow+\infty} L_{1}(t)=-\infty .
$$

Next, $L_{1}(t)=0$ iff

$$
\underbrace{\cosh \log \left(2 \sinh \frac{\pi t}{6}\right)}_{\geqslant 1}=\frac{1+t^{2}}{\pi} .
$$

Hence, there are no zeros in the interval $\left(0, t_{0}\right)$. The function $L_{1}(t)$ is negative in the interval (cf. (5.3)). Here $t_{0}=\sqrt{\pi-1}=1.463418 \ldots$ By (5.2),

$$
\begin{aligned}
L_{1}(t) & =\log \left(\frac{2}{\pi}\left(1+t^{2}\right) e^{-\frac{\pi t}{6}} \frac{1}{1+\frac{e^{-\frac{2 \pi t}{3}}}{1-e^{-\frac{\pi t}{3}}}}\right)= \\
& =\underbrace{\log \frac{2}{\pi}+\log \left(1+t^{2}\right)-\frac{\pi t}{6}}_{=\Psi_{1}(t)}+\underbrace{\log \left(1-\frac{1}{e^{\frac{2 \pi t}{3}}-e^{\frac{\pi t}{3}}+1}\right)}_{=\Psi_{2}(t)} .
\end{aligned}
$$


The function $\Psi_{1}(t)$ is concave for $t>1$. Indeed, consider the second derivative,

$$
\Psi_{1}^{\prime \prime}(t)=2 \frac{1-t^{2}}{\left(1+t^{2}\right)^{2}}<0 .
$$

Next, consider the second derivative of $\Psi_{2}(t)$,

$$
\Psi_{2}^{\prime \prime}(t)=\frac{-\pi^{2} e^{\frac{2 \pi t}{3}}}{9\left(e^{\frac{2 \pi t}{3}}-e^{\frac{\pi t}{3}}+1\right)^{2}\left(e^{\frac{\pi t}{3}}-1\right)^{2}} \underbrace{\left(4 e^{\frac{2 \pi t}{3}}-7 e^{\frac{\pi t}{3}}+4\right)}_{>0}<0 .
$$

Combining (5.6) and (5.7) we obtain, that the function $L_{1}(t)$ is concave for $t>$ 1. Thus, the concave function on an open set takes negative, then positive (e.g. $L_{1}((6 / \pi) \log 3)>0$ ), then again negative values (cf. (5.3)). Hence, it has unique positive maximum in the interval $\left(t_{1}, t_{2}\right)$. Here $t_{1}$ and $t_{2}$ are the roots of the function $L_{1}(t)$. The values of the roots we obtain numerically with any sufficient accuracy.

Next, let us denote

$$
L_{0}(\sigma)=L(\sigma, 0) .
$$

Lemma 7. For $1 / 2<\sigma<1$, the function $L_{0}(\sigma)$ is negative.

Proof. By (3.8) and (3.15) we have

$$
L_{0}(\sigma)=(2 \sigma-1) \log \frac{2}{\pi} \sigma^{2}+I_{1}(\sigma) .
$$

Calculating the derivative of the function (cf. (3.10)) we obtain

$$
\begin{aligned}
L_{0}^{\prime}(\sigma)= & \underbrace{2 \log \frac{2}{\pi} \sigma^{2}+\frac{2 \sigma-2}{\sigma}}_{=Q_{1}(\sigma)}+\underbrace{\frac{-\pi}{6}(2 \sigma-1) \cot \pi \sigma+\frac{-\pi}{2 \sin \pi \sigma}}_{=Q_{2}(\sigma)}+ \\
& +\underbrace{\frac{4 \pi}{3 \sqrt{3} \frac{1}{1-2 \cos \left(\frac{2 \pi \sigma}{3}-\frac{\pi}{3}\right)}+2} .}_{=Q_{3}(\sigma)}
\end{aligned}
$$

Let us estimate $Q_{k}(\sigma)$ functions from above. For $1 / 2<\sigma<1$ the function $Q_{1}(\sigma)$ is negative since the derivative

$$
Q_{1}^{\prime}(\sigma)=\frac{4}{\sigma}+\frac{2}{\sigma^{2}}>0
$$

and $Q_{1}(1)<0$.

For $1 / 2<\sigma<1$ the function

$$
Q_{2}(\sigma)=\underbrace{\frac{-\pi}{6 \sin \pi \sigma}}_{<0} \underbrace{((2 \sigma-1) \cos \pi \sigma+3)}_{=q_{2}(\sigma)} .
$$


The derivative

$$
q_{2}^{\prime}(\sigma)=2 \cos \pi \sigma-(2 \sigma-1) \pi \sin \pi \sigma<0,
$$

while $q_{2}(1)=2>0$, hence $q_{2}(\sigma)>0$, and $Q_{2}(\sigma)$ is negative.

For $1 / 2<\sigma<1$ the function

$$
Q_{3}(\sigma)=\frac{4 \pi}{3 \sqrt{3}} \frac{1}{1-2 \cos \left(\frac{2 \pi \sigma}{3}-\frac{\pi}{3}\right)}+2<0 .
$$

Hence, $L_{0}^{\prime}(\sigma)<0$ with $L_{0}(1 / 2)=0$, yielding us the statement of the lemma.

\section{Proof of THE THEOREM FOR THE MOdIFIEd SELbERG ZETA-FUNCTION}

Now we can prove the Theorem 3.

Proof. Consider max value of the function $L(\sigma, t)$ in the rectangle $(\sigma, t) \in(1 / 2,1) \times$ $\left(0, t_{1}\right)$. By Lemma 5 , the function $L(\sigma, t)$ has no stationary points in the interior of the rectangle, so it suffices to investigate the behaviour of the function on vertices of the rectangle. By Lemma 4, the function $L(\sigma, t)$ is convex by $\sigma$ and the derivative by $t$ is positive, hence we must consider the first zero of the function $L_{1}(t)$ (cf. Lemma 6). Note that

$$
\lim _{\sigma \rightarrow 1 / 2+} L(\sigma, t)=0 .
$$

Next let us consider max value of the function $L(\sigma, t)$ in the strip $(\sigma, t) \in(1 / 2,1) \times$ $\left(t_{2}, \infty\right)$. By Lemma 5 , function $L(\sigma, t)$ has no stationary points in the interior of the strip, so it suffices to investigate the behaviour of the function on vertices. By Lemma 4, the function $L(\sigma, t)$ is convex by $\sigma$ and the derivative by $t$ is negative, hence we must consider the second zero of the function $L_{1}(t)$ (cf. Lemma 6). By Lemma 7 and (6.1), it gives us $L(\sigma, t)<0$. Consequently (cf. (3.9))

$$
\log \left|\frac{W(s)}{W(1-s)}\right|<0
$$

yielding us the statement of the theorem.

\section{ACKNOWLEDGEMENTS}

Authors would like to thank the anonymous reviewer for careful reading of the manuscript and providing constructive comments and suggestions, which have helped them to improve the quality of the paper.

\section{REFERENCES}

[1] R. D. Dixon and L. Schoenfeld, "The size of the Riemann zeta-function at places symmetric with respect to the point 1/2." Duke Math. J., vol. 33, no. 2, pp. 291-292, 1966, doi: 10.1215/S00127094-66-03333-3.

[2] R. Garunkštis and A. Grigutis, "The size of the Selberg zeta-function at places symmetric with respect to the line $\operatorname{Re}(\mathrm{s})=1 / 2 . "$ Results. Math., vol. 70, no. 1, pp. 271-281, 2016, doi: 10.1007/s00025-015-0486-7. 
[3] H. Iwaniec, Lectures on the Riemann Zeta Function. American Mathematical Society, 2014.

[4] M. Minamide, "On zeros of the derivative of the modified Selberg zeta function for the modular group." J. Ind. Math. Soc., vol. 80, no. 3-4, pp. 275-312, 2013.

[5] H. Rademacher, Topics in analytic number theory. Springer-Verlag Berlin Heidelberg, 1973. doi: 10.1007/978-3-642-80615-5.

Authors' addresses

I. Belovas

Vilnius University, Institute of Mathematics and Informatics, Akademijos str. 4, LT-04812 Vilnius, Lithuania and Vilnius Gediminas Technical University, 10223 Vilnius, Lithuania

E-mail address: Igoris.Belovas@mii.vu.1t

\section{Sakalauskas}

Klaipèda University, LT-92294 Klaipèda, Lithuania

E-mail address: Leonidas. Sakalauskas@mii.vu.lt 